\title{
Comparative Analysis of the Efficiency of Methods for Optimizing the Flow Distribution in Water Supply Systems with a Large Number of Active Sources
}

\author{
Sergey Dyadun ${ }^{1 *}$, Vladlen Kuznetsov ${ }^{2}$, and Valentin Yesilevskyi ${ }^{3}$ \\ ${ }^{1}$ V.N. Karazin National Kharkiv University, Kharkiv, Ukraine; \\ ${ }^{2}$ National University of Science and Technology - Stary Oskol Technological University \\ (MISiS), Belgorod Region, Russia; \\ ${ }^{3}$ National University of Radioelectronics, Kharkiv, Ukraine
}

\begin{abstract}
Optimization of the water flow distribution is an important problem in the field of the operative control over technological processes in water supply systems. In this article we describe the comparative analysis results showing the efficiency of various methods used to optimize flow distribution in water supply systems with a large number of active sources. The research has shown that the Hooke and Jeeves method of the direct search is the most efficient in terms of the cost of computer time and the amount of used memory.
\end{abstract}

\section{Introduction}

Optimization methods used for the flow distribution are sufficiently studied and tested in practice for water supply systems with a small number of active sources [1,2]. However, system developers have to deal with a large number of simultaneously working pumping stations when building operative control systems for water supply systems (WSS) in cities and when designing automatized workstations for water supply network supervisors. The complexity of solving the optimization task for the flow distribution in the WSS increases when the number of cooperating active sources which determine the number of variables in the optimization task is increasing. Numerous studies have been focused on mathematical modeling of WSS and other pipeline systems, such as described in these papers [1-10]. The researches [11-13] consider operative planning for the water supply systems. The operating modes optimization for pumping stations of the WSS is discussed in [14-17].

This article contains a comparative analysis of the efficiency of various optimization methods used to determine the optimal flow distribution in water supply systems with a large number of active sources.

\section{Formulation of the problem}

* Corresponding author: s.v.daulding@gmail.com 
Water supply systems in modern cities belong to the class of large systems operated under conditions of incomplete and unreliable information about the controlled object.

Researches [1-3] offer an approach for the operative control of WSS based on the use of specific for water supply networks dictating points which is widely applied in practice. The basic idea is that the quality of the entire network functioning can be characterized by its state in these points. The dictating point of the WSS is the point at which the pressure value at the current time is minimal. The stochastic nature of water consumption processes causes continuous changes in the flow distribution in the network and the appearance of numerous dictating points. Thus, the main task can be reduced to the synthesis of the control system ensuring a given quality level of the WSS functioning in critical points.

Solving the problem of operational control of flow distribution in water supply systems at each stage - operational planning and stabilization - is usually separated in time and location, requires a different volume, composition and nature of operational information, various mathematical models describing the control object, various criteria and methods for solving control tasks. However, the implementation of each control stage requires solving a certain number of tasks. They can be categorized into modeling tasks that needs to be done outside of the real control loop and control tasks solved in real time.

The steady-state flow distribution model is the most adequate mathematical model of the control object for solving tasks at the stage of operative planning for flow distribution processes in the WSS. The steady-state flow distribution model contains information about the network topology and technological parameters of all elements of the equivalent circuit of the water supply network. Dynamic models are used to solve the task of modes stabilization [1-3]. According to this approach, the operative control process for the flow distribution in WSS is decomposed into two stages: the operative planning for the modes of functioning of the WSS and their stabilization [1-3]. The problem of the operative control of the WSS is formulated as a two-stage nonlinear stochastic programming task [2].

The optimization task for the operating modes of pumping stations when they work together on the network is solved at the upper level of the operational planning stage of functioning modes of WSS. The minimum amount of energy consumption at pumping stations is used as a criterion. The planned values of flow rates and pressures at the outputs of pumping stations, as well as the location of future dictating points of the water supply network where it is necessary to stabilize the planned pressure values are determined as a result of solving this minimization task $[1,2,13]$. Then the optimal structure and operation parameters of pumping stations are determined for each of them at the lower level of the operational mode planning stage to ensure the planned output parameters [14]. Stabilizing pressure in the dictating points of the network allows correcting planning errors by clarifications the pressures at these points.

\section{Mathematical formulation of a problem}

Thus, the task of optimizing the flow distribution in the water supply network holds an important place in the problem of operative control for technological processes of the functioning of water supply systems. Let us consider the formulation of the problem and methods of its solution for the WSS of a city with a large number of pumping stations (PS).

The purpose of this task is to determine such values of the components of the flow rate $\bar{q}_{\text {out }}^{(a)}$ and pressure $\bar{H}_{\text {out }}^{(a)}$ vectors at the output of all pumping stations, which at known predicted values of the nodal flow rates $q_{j}, j \in N$ in the network and performing of all imposed restrictions would ensure the achievement of a maximum of some criterion that characterizes the efficiency of the functioning of the WSS. I.e. it is necessary to distribute 
the load (flow) between the PS in such a way that maximum efficiency is achieved while ensuring the specified quality of water supply to all consumers.

We use an amount of energy consumption at pumping stations as a criterion for the WSS operational efficiency. We denote the set of active elements, trunk network sections and fictitious arcs with consumers as $L, M, N$ respectively; $E=L \cup M \cup N$.

The sets with index 1 correspond to tree branches for the network graph, and those with index 2 correspond to chords. Then the task of optimizing the distribution of the load between PS when they work together on the water supply network has next form

$$
Y\left[\bar{q}_{\text {out }}^{(a)}, \bar{H}_{\text {out }}^{(a)}\right]=\sum_{i \in L} P_{i}^{*}\left(q_{\text {outi }}^{(a)}, H_{\text {outi }}^{(a)}\right) \rightarrow \min _{q_{\text {outi }}^{(a)} \in \Omega}
$$

$\Omega: \quad f_{r}=\operatorname{signq}_{r} r_{r}\left|q_{r}\right|^{2}+\sum_{i \in M_{1}} b_{1 r i} \operatorname{signq}_{i} r_{i}\left|q_{i}\right|^{2}=0, r \in M_{2}$

$$
\begin{gathered}
f_{r}=H_{r h}-H_{j k}+\sum_{i \in M_{1}} b_{1 r i}\left(\operatorname{signq}_{i} r_{i}\left|q_{i}\right|^{2}+h_{i}^{(G)}\right)=0, r \in N, j \in L_{1} \\
f_{r}=H_{j k}-H_{r h}+\sum_{i \in M_{1}} b_{1 r i}\left(\text { signq }_{i} r_{i}\left|q_{i}\right|^{2}+h_{i}^{(G)}\right)=0, r \in L_{2}, j \in L_{1} \\
q_{i}=\sum_{r \in M_{2} U L_{2}} b_{1 r i} q_{r}+Q_{i}^{+}, i \in M_{1} \cup N_{1} \\
q_{\text {outi }}^{(a)+}\left(H_{\text {outi }}^{(a)}\right) \leq q_{\text {outi }}^{(a)} \leq q_{\text {outi }}^{(a)++}\left(H_{\text {outi }}^{(a)}\right), i \in L \\
h_{j} \geq h_{j}^{+}, j \in N,
\end{gathered}
$$

where $\quad P_{i}^{*}(\cdot)=q_{\text {outi }}^{(a) *} H_{\text {outi }}^{(a) *}, i \in L$;

$$
Q_{i}^{+}=\sum_{r \in N} b_{1 r i} q_{r}^{+}=\text {const }
$$

$q_{\text {outi }}^{(a) *}, H_{\text {outi }}^{(a) *}$ are the optimal values of the flow rate and pressure at the outlet of the $i$-th PS; $b_{1 r i}$ is element of the cyclomatic matrix $B_{1}[1] ; q_{i}, i \in E$ is water consumption in the $i$-th arc of the water supply network; $r_{i}, i \in M$ is hydraulic resistance of the $i$-th section of the WSS; $h_{i}^{(G)}, i \in M$ is difference in geodetic heights of the $i$-th section of the WSS; $q_{\text {outi }}^{(a)+}, q_{\text {outi }}^{(a)++}, i \in L$ are lower and upper limits of the flow rate through the PS, which depend on the value $H_{\text {outi }}^{(a)} ; h_{j}, h_{j}^{+}, j \in N$ are the actual and minimum allowable pressure in the j-th node of the WSS, respectively; $H_{r h}, H_{j k}$ are - pressures at the beginning of the $r$-th and at the end of the $j$-th arc, respectively.

\section{Solution methods}

This problem can be solved by methods of nonlinear mathematical programming or search optimization based on the hydraulic calculation of the water supply network. Its specific feature is the algorithmic setting of the target function. Instead of solving a complex problem of mathematical programming (1) - (8), it is advisable in the field

$$
q_{\text {outi }}^{(a)+}\left(H_{\text {outi }}^{(a)}\right) \leq q_{\text {outi }}^{(a)} \leq q_{\text {outi }}^{(a)++}\left(H_{\text {outi }}^{(a)}\right), i \in L
$$




$$
\sum_{i \in L} q_{\text {outi }}^{(a)}=\sum_{j \in N} q_{j}^{(c)}
$$

to look for a point $\bar{q}_{\text {outi }}^{(a)}=\left[q_{\text {outi }}^{(a)}, i \in L\right]$, where after solving the problem of hydraulic calculation, the value of criterion (1) is the smallest.

As the researchs have shown, the function $Y\left[\bar{q}_{\text {out }}^{(a)}, \bar{H}_{\text {out }}^{(a)}\right]$ determined in accordance with expression (1) is always unimodal and convex downward. An interesting property of this problem is that the function $Y[\cdot]$ is not defined outside the valid range. In this case, it is sufficient to replace the values of the objective function at the inadmissible points with a very large positive value, and they will be automatically discarded during the search [18].

The simplest method for solving the problem of optimizing the flow distribution in the WSS is a random search in the domain $\Omega$. For a network for which one active element works, the solution to the problem of hydraulic calculation will also be optimal according to criterion (1). When working on a network of two active sources, this problem is reduced to the problem of one-dimensional search engine optimization. With a larger number of variables, it is necessary to use multivariate optimization methods.

To research the efficiency of solving the problem of optimizing the flow distribution in the WSS, we will also use the most effective and common methods: coordinate descent; scanning with variable step; deformable Nelder-Mead polytope; direct search for Hook and Jeeves; Rosenbrock; Powell [18-20]. Since the goal function is set algorithmically, it is not possible to obtain analytical expressions for its derivatives. Therefore, the use of higher order multivariate optimization methods is not possible.

\section{Research results}

When comparing these methods, we used computer time costs required to achieve convergence of algorithms and the amount of PC memory occupied as efficiency criteria. The investigations were carried out on the basis of a model of a real WSS, consisting of 382 network arcs and 10 active sources. In the simulation, the number of active sources working together on the water supply network varied within $3 \leq l \leq 10$, respectively, the number of independent variables $l-1$. In terms of the amount of PC memory occupied, the differences turned out to be insignificant. They are mainly determined by the number of variables $l$ of the function to be minimized, while arrays of much higher dimension are used to calculate the target function. In this way, we will take as the main criterion of the minimum of consumption of computer time. The scanning method with a variable step is the simplest to implement and allows you to always find a global extremum with a sufficiently dense arrangement of the studied points. However, in spite of its reliability, it turns out to be ineffective due to the extremely large number of calculations of the function, which sharply increases with increasing number of variables. A disadvantage of the coordinate descent method is the significant dependence of its convergence rate on the choice of the coordinate system. The deformable polytope method is not reliable enough. Hook and Jeeves' method works on ridges, its disadvantage is that it can skip the ridge. There are other minimization methods that do not require calculating the derivatives of the objective function. But most of them use difference approximations of partial derivatives of functions $f(x)$, i.e. are essentially variants of gradient methods.

The results of numerical analysis when working on a network of three active sources 
indicates good convergence of all methods with a relatively small number of iterations $(k<30)$. The deformable polyhedron method is somewhat inferior to other methods. The coordinate descent method quickly converges at the first stage of determining the direction of minimization, then when the methods of one-dimensional minimization in the direction are used, its effectiveness decreases. During the analysis, several methods were used as a minimization procedure in the direction (the golden section method, the Fibonacci method, the calculation of the minimum using a quadratic approximation with the localization of the minimum point, the dichotomy method). They all give almost the same convergence.

The efficiency of the algorithms for optimizing the flow distribution in the WSS depends significantly on the dimension of task, i.e. on the number $l-1$ of independent variables. In some of the considered methods, several function evaluations are performed at each iteration. Table 1 shows the number of calculations of the function required to achieve a relative calculation error of $0.005 \%$ for a various number of variables.

Table 1. Number of function calculations

\begin{tabular}{|l|c|c|c|}
\hline \multirow{2}{*}{ Method } & \multicolumn{3}{|c|}{ Number of variables } \\
\cline { 2 - 4 } & 2 & 5 & 9 \\
\hline Direct search for Hook and Jeeves & 23 & 136 & 230 \\
\hline Coordinate descent & 22 & 498 & 1000 \\
\hline Deformable Nelder-Mead polytope & 29 & 1000 & 1000 \\
\hline Rosenbrock & 17 & 174 & 348 \\
\hline Powell & 18 & 209 & 303 \\
\hline
\end{tabular}

The advantages of the Hook and Jeeves method are clear. Even for 9 variables, it gives a convergence rate that is quite acceptable for practical purposes, while when the deformable polytope method was used, the required degree of accuracy was not achieved and with 1000 calculations of the function even for 5 variables. The convergence of the coordinate descent method also deteriorates with an increase in the number of variables. This is due to the fact that at each iteration there are $\mathrm{n}$ calls to the subroutine for calculating the minimum of function in direction that requires up to 10 calculations of the function (although the number of iterations in the Hook and Jeeves method and the coordinate descent method does not differ significantly).

Table 2 shows the number of iterations required to achieve a given accuracy of convergence of the considered algorithms for different number of optimization variables.

Table 2. Number of iterations

\begin{tabular}{|l|c|c|}
\hline \multirow{2}{*}{ Method } & \multicolumn{2}{c|}{ Number of variables } \\
\cline { 2 - 3 } & 2 & 5 \\
\hline Direct search for Hook and Jeeves & $2-3$ & $10-12$ \\
\hline Coordinate descent & $2-3$ & $10-12$ \\
\hline Deformable Nelder-Mead polytope & $15-17$ & 100 \\
\hline Rosenbrock & $2-3$ & $10-15$ \\
\hline Powell & $2-3$ & $10-15$ \\
\hline
\end{tabular}

\section{Conclusions}

Thus, when solving problems of optimizing the flow distribution in water supply systems of large dimensions (the number of active sources operating on the network, $3 \leq l \leq 10$ ), the method of direct search for Hook and Jeeves is the most efficient in terms of the cost of computer time and the occupied volume of PC memory. 
It is advisable to use the results obtained in the development and operation of operational control systems for technological processes of the functioning of WSS in large cities, automatized workstations for dispatchers of WSS, automatized design systems for urban water supply networks to determine the optimal modes of functioning of WSS.

\section{References}

1. A.G. Evdokimov, A.D. Tevyashev, Operational control of the flow distribution in engineering networks, 144 (1980) [in Russian]

2. A.G. Evdokimov, A.D. Tevyashev, V.V. Dubrovskiy, Modeling and optimization of the flow distribution in engineering networks, 368 (Moscow, Stroyizdat, 1990) [in Russian]

3. A.G. Evdokimov, A.D. Tevyashev, V.V. Dubrovskiy, Streaming distribution in engineering networks, 199 (Moscow, Stroyizdat, 1979) [in Russian]

4. A.G. Evdokimov, Optimal problems on the engineering networks, 153 (Kharkov, Vishcha Shkola, 1976) [in Russian]

5. F. Fallside, P.F. Perry, R.H. Burch, K.C. Marlow, Computer Simulation of Water Resourses Systems, 12, 617 (1975)

6. A.P. Merenkov, V.Y. Hasilev, Theory of hydraulic circuits, 279 (Moscow, Nauka, 1985) [in Russian]

7. N.N. Novitsky, M.G. Suharev, A.D. Tevyashev, et al., Energy Pipeline System: methodological and applied problems of mathematical modeling (Novosibirsk, Science, 2015) [in Russian]

8. N.N. Abramov, Theory and Methodology of Calculation of Water Supply and Distribution Systems (Moscow, Stroyizdat, 1985) [in Russian]

9. N.N. Novitskii, O.V. Vanteyeva, Chaotic Modeling and Simulation (CMSIM), 1, 95 (2014)

10. S.V. Dyadun, RadioElectronics \& Informatics Journal, 4, 54 (2000)

11. A.D. Tevyashev, O.I. Matvienko, Econtechmod. An International Quarterly Journal, 3(3), 61 (2014)

12. J. Burgschweiger, B.B. Gnadig, M.C. Steinbach, Nonlinear programming techniques for operative planning in large drinking water networks. In: Konrad-Zuse-Zentrum fur Informationstechnik Berlin. - Berlin: ZIB-Report (2005)

13. S.V. Dyadun, RadioElectronics \& Informatics Journal, 20, 111 (2002) [in Russian]

14. S.V. Dyadun, City utilities, Tehnika, 1, 63 (1992)

15. C. Reinbold, V. Hart, Florida Water Resources Journal, 44 (2011)

16. I. Pulido-Calvo, J.C. Gutiérrez-Estrada, Environmental Research Journal, Nova Science Publishers, 5 (3), 1 (2011)

17. B. Lipták, Control Promoting Excellence in Process Automation, 12 (2009)

18. A.G. Evdokimov, Minimization of functions and its application to the tasks of automatized control of engineering networks (Kharkov, 1985) [in Russian]

19. L.A. Rastrigin, Extreme control systems (Moscow, 1974) [in Russian]

20. D. Himmelblau, Applied nonlinear programming (Moscow, 1975) [in Russian] 